\title{
Lunch at school and children's cognitive functioning in the early afternoon: results from the Cognition Intervention Study Dortmund Continued (CoCo)
}

\author{
Maike Schröder $^{1 *}$, Katrin Müller ${ }^{2}$, Michael Falkenstein ${ }^{3}$, Peter Stehle ${ }^{4}$, Mathilde Kersting ${ }^{1}$ and Lars Libuda ${ }^{5,6}$ \\ ${ }^{1}$ Research Institute of Child Nutrition (FKE) Dortmund, University of Bonn, Heinstück 111, 44225 Dortmund, Germany \\ ${ }^{2}$ Institute for Prevention and Public Health, University of Applied Sciences (DHfPG), Herrmann Neuberger Sportschule 3, \\ 66123 Saarbrücken, Germany \\ ${ }^{3}$ Institute of Working, Aging and Learning (ALA), Hiltroper Landwehr 136, 44805 Bochum, Germany \\ ${ }^{4}$ Department of Nutrition and Food Sciences, Nutritional Physiology, University of Bonn, Endenicher Allee 11-13, 53115 \\ Bonn, Germany \\ ${ }^{5}$ Department of Child and Adolescent Psychiatry, University Hospital Essen, University of Duisburg-Essen, Virchowstr. 174 , \\ 45147 Essen, Germany \\ ${ }^{6}$ Department of Pediatrics, Marien-Hospital Wesel, Research Institute for the Prevention of Allergies and Respiratory Diseases in \\ Childhood, Pastor-Janßen-Str. 8-38, 46483 Wesel, Germany
}

(Submitted 28 January 2016 - Final revision received 20 May 2016 - Accepted 7 July 2016 - First published online 9 September 2016)

\begin{abstract}
Studies about effects of school lunch on children's cognition are rare; two previous studies (CogniDo, CogniDo PLUS) generally found no negative effects of lunch on children's cognitive performance at the end of lunch break (i.e. $45 \mathrm{~min}$ after finishing lunch), but suggested potential beneficial effects for single parameters. Therefore, the present study investigated the hypothesis of potential positive effects of school lunch on cognitive performance at early afternoon $(90 \mathrm{~min}$ after finishing lunch). A randomised, cross-over intervention trial was conducted at a comprehensive school with fifth and sixth grade students. Participants were randomised into two groups: On day 1, group 1 did not eat lunch, whereas group 2 received lunch ad libitum. On day 2 (1 week later), group 2 did not eat lunch and group 1 received lunch ad libitum. The cognitive parameters task switching, working memory updating and alertness were tested using a computerised test battery 90 min after finishing the meal. Of the 204 recruited children, fifty were excluded because of deviations from the study protocol or absence on one of the 2 test days, which resulted in 154 participants. Data showed no significant effects of lunch on task switching, working memory updating and alertness $(P$ values between $0 \cdot 07$ and 0.79). The present study suggests that school lunch does not seem to have beneficial effects on children's cognitive functions regarding the conducted tests at early afternoon. Together with our previous studies, we conclude that school lunch in general has no negative effects on cognitive performance in children. However, beneficial effects seem to be restricted to a relatively short time period after eating lunch.
\end{abstract}

Key words: Children: Cognitive functions: Lunch: Cognition

Considering the extension of all-day schools in Europe, knowledge about potential effects of school lunch on children's cognition is becoming increasingly important. Short-term lunch effects on cognition were primarily examined in adults with equivocal results, until now. Although sustained attention and the ability to discriminate have been shown to be worsened after lunch ${ }^{(1,2)}$, other cognitive aspects were improved (reading ability) ${ }^{(3)}$ or did not change (selective attention) ${ }^{(4)}$. One explanation for negative lunch effects on cognitive performance is the post-lunch dip - a naturally occurring nadir in performance at midday. Studies in adults have shown that this dip is worsened by lunch ${ }^{(5,1)}$. Therefore, it could be hypothesised that skipping lunch could result in an alleviation or prevention of this post-lunch dip. However, these results were obtained in adults and are not necessarily transferable to children due to constitutional and metabolic differences between children and adults (e.g. still rapid brain growth, high metabolic rate in children). Until now, studies in children about the effects of meals on cognition have mainly concentrated on breakfast ${ }^{(())}$. A body of research work has shown short-term benefits for cognitive performance when children eat breakfast instead of skipping it ${ }^{(6)}$. However, other studies showed that poorly nourished children benefit more than well-nourished children ${ }^{(7)}$. Although no definitive conclusions can be drawn about short-term benefits of breakfast for cognitive performance in children in general, there is at least a noticeable indication for a beneficial influence ${ }^{(8,6,9)}$.

Studies about school lunch and short-term effects on children's cognition are rare ${ }^{(10)}$. To the best of our knowledge, there are only two cross-over, intervention trials from our group, which

Abbreviations: $\mathrm{CF}$, cognitive functions; CoCo, Cognition Intervention Study Dortmund Continued; L, lunch; NL, no lunch.

* Corresponding author: M. Schröder, fax +49 23171 1581, email schroeder@fke-do.de 
provided first insights into the impact of school lunch on cognitive performance in children at the end of lunch break (CogniDo ${ }^{(11)}$, CogniDo PLUS ${ }^{(12)}$ study). Both studies did not reveal negative effects of lunch on several parameters of cognitive functions (CF) (i.e. task switching, working memory updating, inhibition, alertness, selective attention, block span) when determined about $45 \mathrm{~min}$ after finishing lunch. For single parameters such as omission errors in the alertness task and false alarms in the updating task (regarding the working memory) results even point to beneficial effects of lunch at the end of lunch break. Even though no lunch-related cognitive impairment was observed in these two studies, it has to be considered that a post-lunch dip in children could appear as the afternoon progresses. In adults, a post-lunch dip was observed about 60-120 min after lunch ${ }^{(1,4,13)}$, indicating that the time span between a meal and the measurement of cognitive performance could have a relevant influence ${ }^{(13)}$.

Therefore, the objective of the Cognition Intervention Study Dortmund Continued (CoCo) was to investigate the potential positive effects of school lunch on cognitive performance in children at early afternoon (90 min after finishing lunch instead of $45 \mathrm{~min}$ in the previous intervention studies). In order to provide comparability with both previous studies, cognitive tests that proved to be the most sensitive were chosen - that is, the alertness task from the first study (CogniDo) ${ }^{(11)}$ and task switching and working memory updating task from the second study (CogniDo PLUS) ${ }^{(12)}$. On the basis of the previous results of these two studies, we hypothesise that children will perform better on lunch day than on no lunch day.

\section{Methods}

\section{Study design and participants}

Similar to the previous studies, the CoCo study was conducted as a randomised, open-label, $2 \times 2$ cross-over intervention trial. The same all-day comprehensive school in Gelsenkirchen, Germany, was chosen for the experiment. In total, the field period spanned 19 weeks between October 2014 and March 2015 including 3 weeks of holidays. Each subject had to participate on two study days with 1 week in between on the same weekday.

The participants were recruited from the fifth and sixth grades (twelve classes). The students of the sixth grade in the present study had already participated in the previous CogniDo PLUS study (as fifth grade students). Children with diseases with potential consequences of fasting and children on special diets, who were not allowed to eat the study meal, were excluded from participation. Children with a diagnosed learning disorder reported by the class teacher were allowed to participate, but were excluded post hoc for the analyses. Out of 324 students, 204 provided informed written consent to participate. A cluster randomisation per class with a block size of four participants was conducted to assign participants to one of two study groups: on day 1 of the study, group 1 did not eat lunch (no lunch, NL), whereas group 2 received lunch ad libitum (lunch, L); 1 week later, on day 2 of the study, group 1 was in the L condition and group 2 in the NL condition. All children, who participated on both intervention days, received a ball as reward for their participation.

The study was approved by the Ethics Committee of the University of Bonn and registered at clinicaltrials.gov (NCT02344056). All assessments were made in compliance with the Declaration of Helsinki.

\section{Study schedule}

The study design was integrated in the regular school routine and corresponded to the study schedules of the previous studies (CogniDo ${ }^{(11)}$, CogniDo PLUS ${ }^{(12)}$ ), but differed in the time interval between lunch break and assessment of cognitive performance (Fig. 1).

On both test days, a standardised breakfast (wholemeal bread with margarine, poultry salami or Gouda cheese and carrot sticks) ad libitum was offered to the students in the regular morning break at 09.15 hours. During the regular lunch break, starting at 12.25 hours, the subjects either received lunch ad libitum (pasta with or without Bolognese sauce as desired) and an optional apple in the school canteen prepared as usual by the kitchen staff (L), or the subjects skipped lunch and stayed in a separate room (NL). Water was available at any time in both test situations. The amount of pasta was individually weighed before and after the meal $\pm 5 \mathrm{~g}$. After lunch, all students (L and NL) had their common break (until 13.20 hours) and the regular seventh lesson (13.25-14.10 hours). At the beginning of the eighth lesson (14.15 hours), the assessment of

Lunch day

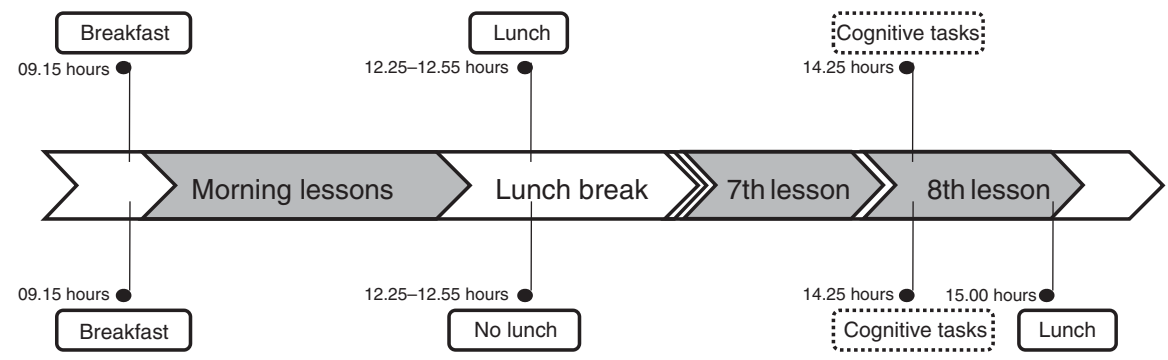

No lunch day

Fig. 1. Schedule of the intervention day and timing for lunch group and no lunch group in the Cognition Intervention Study Dortmund Continued (CoCo) study. 
cognitive performance took place in the school's computer room at about 14.25 hours. After completing these tests (about 15.00 hours), participants in the NL condition received their lunch.

Between the morning break and the lunch break (09.3512.25 hours), all participants were asked to refrain from eating and drinking (except for water and unsweetened tea). The NL group was additionally asked to refrain from eating and drinking until the end of the test day (15.00 hours). In order to assess compliance with the study protocol, the study staff supervised the children in the schoolyard and classrooms during the breaks. In addition, the participants filled out a questionnaire regarding their food and beverage consumption at the end of each intervention day.

\section{Cognitive assessment}

For the assessment of $\mathrm{CF}$, a computerised test battery consisting of three tasks (ALA Institute) was used. Before starting, students had to pass a training phase with a task-by-task explanation by the study personnel and a short practise period. After this training and a 5-min break with low physical exercise, the actual cognitive testing began. Subjects were requested to respond as quickly as possible without sacrificing accuracy. The cognition tasks were applied in the following order: task switching, working memory updating and tonic alertness.

After finishing the cognitive testing for two-thirds of the study sample, the school laptops had to be replaced by desktop computers for school intern reasons. The remaining participants were tested on the new computers. Consequently, the participants were either tested on the laptops or on the desktops. Therefore, calibration of the new computer was not necessary as the analyses of the intervention effects considered the individual differences between two tests days.

Task switching. With an alternative version of the trail making task, subjects' ability to switch between two tasks was measured. This task was presented in three sections: the first two sections (section 1: numbers, section 2: letters) in a non-switch condition, and the third section in switch condition (letters and numbers mixed).

First section - numbers: black numbers from 1 to 26 in white squares were presented in an irregular order on a black computer screen (Fig. 2). The children were asked to click the numbers in an ascending order with the mouse curser. The square with the number 1 was marked green as the starting point. The squares turn green after a correct answer and red after a false answer as a form of feedback. Correctly processed squares fade out. The maximum time limit to finish the task was $3 \mathrm{~min}$.

Second section - letters: this section had the same format as the numbers section, but used letters from $\mathrm{A}$ to $\mathrm{Z}$ instead of numbers (Fig. 3).

Third section - switch: the twenty-six squares contained numbers from 1 to 13 and letters from A to M (Fig. 4). The children were asked to alternately click numbers and letters in ascending order (i.e. 1-A-2-B-3-C...). (a)

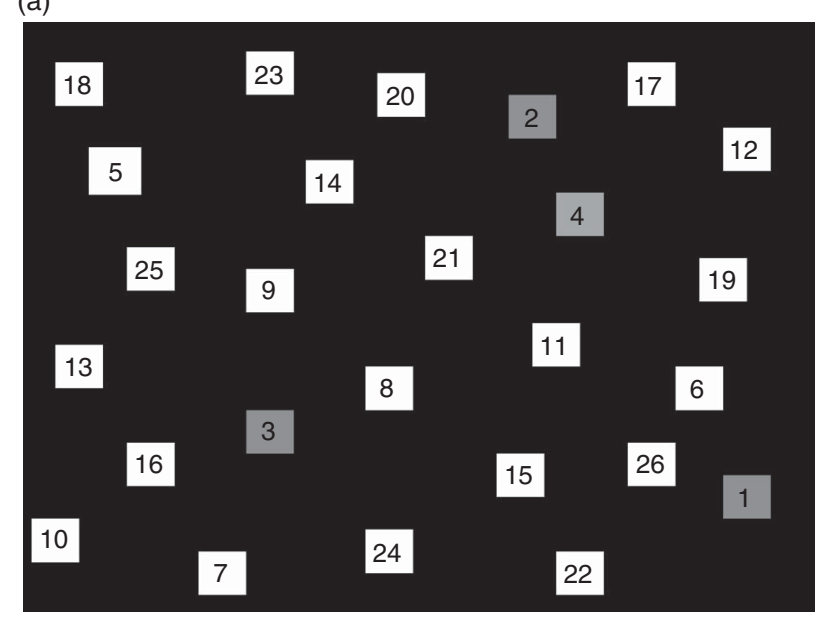

(b)

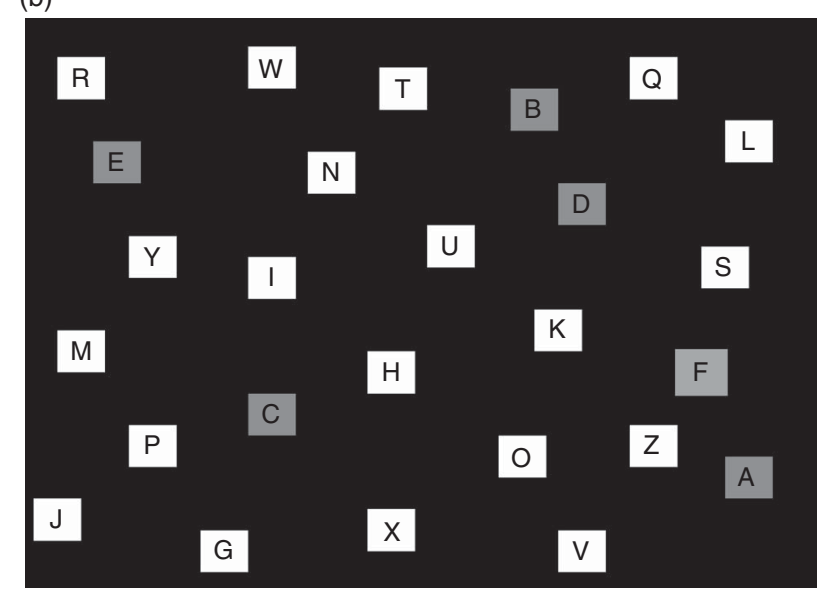

(c)

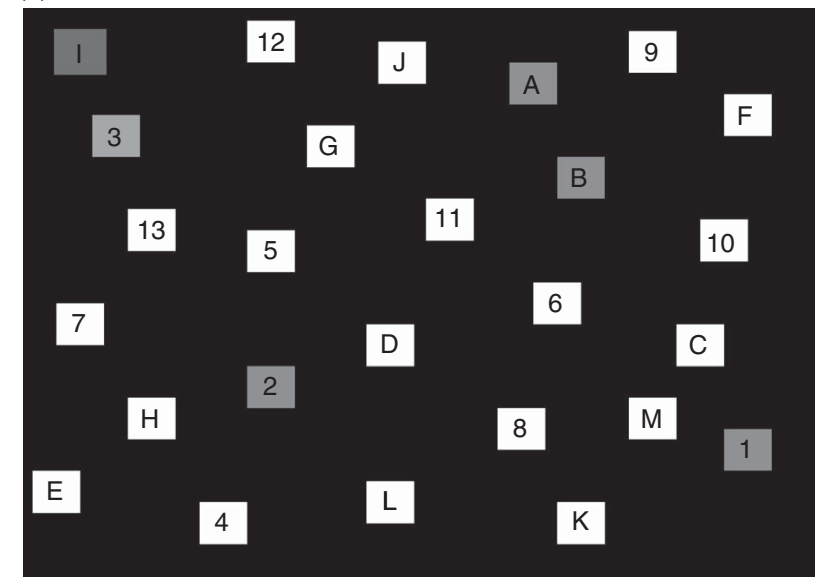

Fig. 2. Cognitive assessment: switch task was presented in three sections the first two sections (section 1: numbers, section 2: letters) in a non-switch condition and the third section in a switch condition (letters and numbers mixed). Screenshots of the sections. (a) First section, numbers, non-switch: children were asked to click the numbers in ascending order with the mouse curser. (b) Second section, letters, non-switch: same format as the first section, but used letters from $A$ to $Z$ instead of numbers. (c) Third section, switch: children were asked to alternately click numbers and letters in ascending order (i.e. 1-A-2-B-3-C...). 


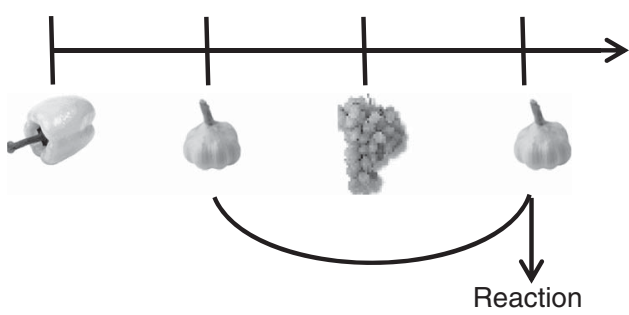

Fig. 3. Cognitive assessment: two-back task to asses working memory updating. Scheme of the task.

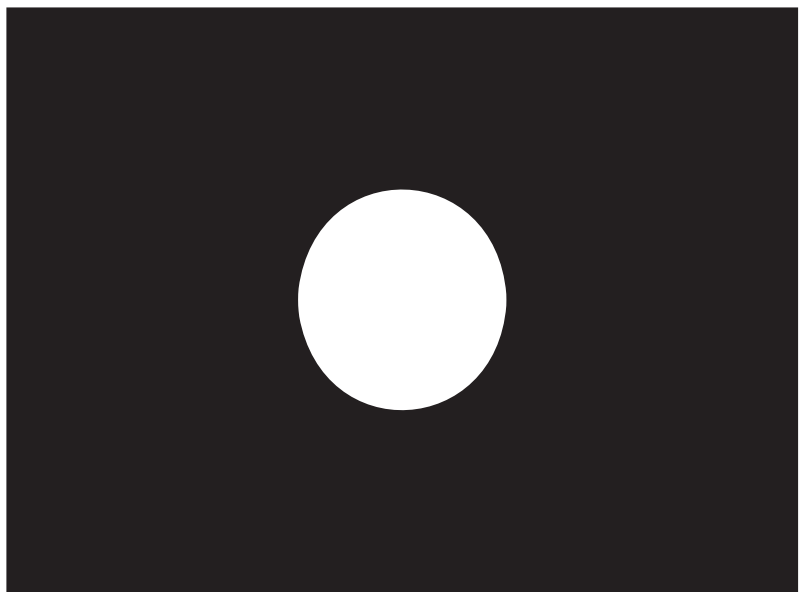

Fig. 4. Cognitive assessment: alertness. Children were instructed to press a predefined key as soon as the white circle appears on the screen.

The outcomes were total reaction time for numbers (for items 2-26), total reaction time for letters (for items 2-13) and switchcosts - that is, the processing time of the third section (switch; items 2-26) minus the first section (numbers; non-switch; items 2-26) minus the difference between the first twelve items of the second section (letter; non-switch; items 2-13) and the first twelve items of the first section (numbers; non-switch; items 2-13). To eliminate implausible data, we excluded all subjects with negative switch costs.

Working memory updating (two-back task). In order to assess the function of constant monitoring and adding or deleting of working memory contents, we used the $n$-back task in a two-back condition. Participants were asked to monitor a sequence of 106 consecutive trials (pictures of fruits and vegetables) presented in a white square in the middle of a black screen. When the current picture matched the picture presented two trials before $(n-2)$, the participant was instructed to press a predefined key on the computer keyboard with the index finger. The stimuli were presented for $500 \mathrm{~ms}$ with an interstimulus interval of $2100 \mathrm{~ms}$ and a maximal reaction time of $1400 \mathrm{~ms}$. No feedback was given. Of the 106 pictures shown, twenty-one were targets (same picture as two trials before)

The outcome variables were the ratio of missings (no reaction while reaction was required), the ratio of false alarms (reaction while no reaction was required) and the mean reaction time while reaction was required. Plausible measurements were defined as ratios of missing $\leq 50 \%$, false alarms $\leq 50 \%$ and reaction times, between quartile 1 minus 1.5 times the interquartile range (IQR) and quartile 3 plus 1.5 times the IQR (i.e. $\geq 196.935$ and $\leq 850.975 \mathrm{~ms}$ ).

Tonic alertness. To measure the level of tonic alertness, we used a simple reaction task. A white fixation cross was presented in the middle of a black screen. In a response stimulus interval of $3300 \mathrm{~ms}( \pm 20 \%)$, a circle followed the cross and the subjects were supposed to press a predefined button as soon as the circle appears (maximal reaction time $1500 \mathrm{~ms}$ ). The test included fifty items.

The outcome variables were the mean reaction time (ms), the deviation of reaction time (ms), the number of omission errors (no reaction after appearance of the circle within $1500 \mathrm{~ms}$ ) and the number of commission errors (reactions during the presence of the fixation cross). Plausible measurements were defined as reaction times $\geq 140.9$ and $\leq 492.58 \mathrm{~ms}$.

\section{Statistical analyses}

All analyses were performed using the statistical software package SAS 9.2 (SAS Institute). $P<0.05$ was considered as statistically significant.

Before conducting the statistical analyses, the raw data of all cognitive tasks were checked for plausibility. With regard to reaction times (working memory updating and alertness), plausible data were defined as values within the range of quartile 1 minus 1.5 times the IQR and quartile 3 plus 1.5 times the $\mathrm{IQR}^{(14)}$. For the 'ratios of false alarms/missings', a predefined limit of $<50 \%$ error rate was defined as plausible as the expected error rate would be $50 \%$ if a subject is choosing his/her reaction completely in random. Therefore, exclusion of error ratios $\geq 50 \%$ was intended to reduce the risk of a systematic error in observations from single subjects as these values indicate a high risk that the task instructions were misunderstood (e.g. if a subject always pushed a button when no reaction was attended). Only plausible data were included in the analysis.

The parameters of the three cognitive tasks were used as outcome variables. All outcome variables were interval scaled. As recommended by Grizzle ${ }^{(15)}$, the sums of the two individual values of the particular outcomes variables of test days 1 and 2 were compared between groups using an unpaired $t$ test for normally distributed data (including normal distribution after transformation) and the Wilcoxon's ranked sum test for nonnormally distributed data to examine potential carry-over effects. Non-normally distributed outcomes were transformed (log, square, root or reciprocal transformation) and analysed using unpaired $t$ test. If transformation did not result in normally distributed parameters, the non-parametric Wilcoxon's ranked sum test was used to analyse carry-over or intervention effects. As no carry-over effects were observed, results from both days were considered for the calculation of the treatment effect.

In addition, linear regression analyses between the lunch size and the change in cognitive performance parameters 
Table 1. Linear regression of lunch weight on the change of main outcome variables of cognitive performance in schoolchildren (10-12 years) participating in the Cognition Intervention Study Dortmund Continued (adjusted for sex and age; only valid models presented)

\begin{tabular}{llrrr}
\hline & & & \multicolumn{2}{c}{ Lunch weight } \\
\cline { 3 - 5 } Tasks & Outcome & Intercept & $\beta$-value & $P$ \\
\hline Switch & Switch costs & -6754.0 & 10.966 & 0.226 \\
& Visual search of letters* & 3635.7 & -9.344 & 0.029 \\
Two-back & Ratio of missings & 23.3 & -0.002 & 0.835 \\
& Reaction time & 4.3 & 0.004 & 0.947 \\
\hline
\end{tabular}

* First twelve reactions.

Table 2. Characteristics of the schoolchildren participating in the Cognition Intervention Study Dortmund Continued (Numbers and percentages; means and standard deviations; medians and 25th/75th percentiles)

\begin{tabular}{|c|c|c|c|c|c|}
\hline & \multicolumn{2}{|c|}{ L-NL ( $n$ 72) } & \multicolumn{2}{|c|}{ NL-L $(n$ 82) } & \multirow[b]{2}{*}{$P$} \\
\hline & $n$ & $\%$ & $n$ & $\%$ & \\
\hline Age (years) & \multirow{2}{*}{\multicolumn{2}{|c|}{$11 \cdot 3$}} & & & $0.31 \dagger$ \\
\hline Mean & & & \multicolumn{2}{|c|}{11.4} & \\
\hline $\mathrm{SD}$ & \multicolumn{2}{|c|}{0.7} & \multicolumn{2}{|c|}{0.6} & \\
\hline Female & 36 & 50 & 31 & 37.8 & $0.13 \ddagger$ \\
\hline Regular lunch§ \| & 63 & $88 \cdot 7$ & 78 & $95 \cdot 1$ & $0.23 \ddagger$ \\
\hline Meal consumption (g) & & & & & $0.87 \ddagger$ \\
\hline Median & \multicolumn{2}{|c|}{360} & \multirow{2}{*}{\multicolumn{2}{|c|}{375}} & \\
\hline 25th/75th percentiles & \multicolumn{2}{|c|}{$275 \cdot 0 / 525 \cdot 0$} & & & \\
\hline
\end{tabular}

L, lunch day; NL, no lunch day.

* Group NL-L skipped lunch during the first period, group L-NL skipped lunch during the second period. $\dagger$ Two sample $t$ test.

$\ddagger \chi^{2}$ Test/Fisher's exact test.

$\S$ Defined as consuming lunch at the school refectory regularly by subscription.

|| Missing data from one subject.

(performance on lunch day and performance on no lunch day) were conducted for all parameters, including age and sex as additional covariables. For all parameters of the alertness task, for the parameter visual search of numbers in the task-switching task and the parameter ratio of false alarms (two-back task), the linear regression revealed non-normally distributed residuals (the association with lunch size was not significant for all of these parameters). As the linear regression might not be meaningful for these parameters, we decided not to present these results in (Table 1).

\section{Results}

\section{Participants}

Of the 204 included participants, nineteen participants (one class) had to be excluded because of a 30-min delay of lunch on their 2 nd test day. In addition, thirty-one children were absent on one of the 2 test days, resulting in 154 participants with complete data. Characteristics of the sample stratified by study groups L-NL ( $n$ 72), that is, having lunch on the 1st test day and skipping it on the 2nd test day, and NL-L ( $n$ 82) are shown in Table 2 . There were no differences in age, sex or consumed amounts of the study meal between both groups. The majority of subjects regularly ate lunch at the school refectory $(88.7 \%$ in L-NL and $95.1 \%$ in NL-L) with no significant difference between the groups $(P=0 \cdot 23)$.

\section{Lunch effects on cognitive functions}

Statistical analyses revealed no significant effects of lunch on the examined CF of task switching, working memory updating (two-back task) and alertness ( $P$ between 0.12 and 0.79 , Table 3). The time for visual search of letters on the taskswitching task showed a trend to slightly increase after having lunch $(P=0 \cdot 07)$. After additional exclusion of subjects who did not follow the study protocol ( $n 67$, per protocol analysis), this trend disappeared (data not shown).

The linear regression analysis revealed a significant negative association between the lunch size and the change in visual search of letters $(\beta=-9.3, P=0.03$ adjusted) in the task-switching task.

\section{Discussion}

The present study revealed no evidence for a lunch-related improvement or decline of cognitive performance in schoolchildren in the early afternoon, about 90 min after finishing lunch. Although our previous studies CogniDo ${ }^{(11)}$ and CogniDo PLUS ${ }^{(12)}$ suggested slight improvements of single cognitive parameters shortly after lunch, the current study did not prove our hypothesis of potentially beneficial cognitive effects of lunch in the afternoon. Interestingly, the linear regression even indicated beneficial effects of lunch size as the individual change between lunch day and no lunch day decreased with 
Table 3. Effects of no lunch $v$. lunch on cognitive performance in schoolchildren (10-12 years) participating in the Cognition Intervention Study Dortmund Continued

(Medians and 25th and 75th percentiles)

\begin{tabular}{|c|c|c|c|c|c|c|c|c|}
\hline \multirow[b]{2}{*}{ Tasks } & \multirow[b]{2}{*}{ Main outcome } & \multicolumn{3}{|c|}{ No lunch } & \multicolumn{3}{|c|}{ Lunch } & \multirow[b]{2}{*}{$P$} \\
\hline & & Median & 25th & 75th & Median & 25th & 75th & \\
\hline \multirow[t]{3}{*}{ Switch ( $n$ 139) } & Switch costs $(\mathrm{ms})^{\star}$ & 23928 & 14990 & 38298 & 21475 & 13367 & 31864 & $0.26 \dagger$ \\
\hline & Visual search letters (ms) $\ddagger$ & 26255 & 22579 & 31270 & 26958 & 22927 & 33928 & $0.07 \dagger$ \\
\hline & Visual search numbers (ms) & 43967 & 38007 & 50548 & 43854 & 38220 & 50782 & $0.36 \dagger$ \\
\hline \multirow[t]{3}{*}{ Two-back ( $n$ 87) } & Ratio of missings (\%) & 28.6 & 19.0 & 35.0 & 28.6 & $19 \cdot 0$ & $38 \cdot 1$ & $0.25 \dagger$ \\
\hline & Ratio of false alarms (\%) & $7 \cdot 1$ & 2.4 & $17 \cdot 6$ & $7 \cdot 1$ & 3.5 & $14 \cdot 1$ & $0.63 \dagger$ \\
\hline & $\mathrm{RT}(\mathrm{ms})$ & $519 \cdot 0$ & $445 \cdot 8$ & 584.8 & 518.5 & $457 \cdot 3$ & $615 \cdot 1$ & $0.36 \dagger$ \\
\hline \multirow[t]{4}{*}{ Alertness ( $n$ 148) } & Mean RT (ms) & 306.0 & 273.1 & 343.9 & 314.9 & $270 \cdot 9$ & 357.0 & $0.12 \dagger$ \\
\hline & Deviation of RT (ms) & $117 \cdot 7$ & 92.5 & $173 \cdot 2$ & $128 \cdot 6$ & 91.4 & 179.7 & $0.53 \dagger$ \\
\hline & Count of omission errors $(n)$ & 0 & 0 & 1 & 0 & 0 & 1 & $0.75 \dagger$ \\
\hline & Count of commission errors $(n)$ & 3 & 1 & 5 & 2 & 1 & 5 & $0.79 \dagger$ \\
\hline
\end{tabular}

$\mathrm{RT}$, reaction time.

* Switch costs = (mean RT switch task) - (mean RT number task) - (mean RT first twelve reactions of letter task-mean RT first twelve reactions of number task).

$\dagger$ Wilcoxon's ranked sum test.

‡ First twelve reactions.

larger lunch sizes for the parameter visual search letters. However, more information was needed for interpreting this result - for example, deviation from regular individual lunch size. It was not possible to obtain this information because of organisational reasons, but this question would be interesting for future studies.

In the earlier CogniDo study ${ }^{(11)}$ ( $n$ 105), the participants made significantly more omission errors in the tonic alertness task on the no lunch day compared with the lunch day $(P=0 \cdot 03)$. The CogniDo PLUS study ${ }^{(12)}$ ( $n$ 195) suggested slightly lower levels of false alarms in the task regarding working memory updating when lunch was eaten compared with the test condition in which lunch was omitted $(P=0.01)-$ that is, after eating lunch, children responded less frequently to non-targets, to which they should not have responded. Both tasks (tonic alertness and working memory updating) of the previous studies were also conducted in the present study without any hints at beneficial effects. A potential explanation for these divergent study results could be the difference in the time span between lunch and cognitive testing, which was about $45 \mathrm{~min}$ after finishing lunch in the former studies, but twice as long with $90 \mathrm{~min}$ in the current study. A comparison between the variable values of the present study and the previous CogniDo and CogniDo PLUS studies shows that the values are located in the same data range. Even though the data were not conducted from the same probands, they seem to be comparable. Considering these results, it might be hypothesised that children's cognitive performance may slightly increase immediately after lunch and may not improve when the fasting period is extended into the early afternoon.

Although the reasons for differences in lunch effects depending on the interval until cognitive testing could not be examined in our studies, one plausible explanation may be the course of blood glucose levels. Glucose levels increase in the early postprandial period, but might have been on a decrease at the time when cognitive performance was tested in the present CoCo study. It could be speculated that an increase in blood glucose is beneficial for cognitive performance, whereas a decrease in glucose might attenuate this effect despite higher levels than in the fasting condition. Studies that investigated glucose uptake showed that the resulting increase in blood glucose levels enhances $\mathrm{CF}$ such as memory $^{(16,17)}$ or sustained attention ${ }^{(18)}$. For example, Owen et $a l .{ }^{(17)}$ demonstrated that a glucose dose of $25 \mathrm{~g}$ enhanced working memory performance following a 2-h fast, and Benton et $a l^{(18)}$ showed that $25 \mathrm{~g}$ glucose as a drink improved sustained attention. However, effects of oral glucose dosage may differ depending on blood glucose resources or the level of depletion of glucose reservoirs, for example, in the liver ${ }^{(17)}$. Furthermore, tests with pure glucose consumption may not simply mirror the effects of a whole meal as applied in our study. Sugars or other carbohydrates as part of a mixed meal increase blood glucose levels more slowly than pure glucose ${ }^{(19,20)}$. Therefore, future studies should assess the role of lunches differing in their glycaemic response on cognitive performance in children and adolescents.

Another influencing factor on cognition could be the postlunch dip phenomenon that may relate to the timing of the meal and the interval until measurements. In adults, a decline in cognitive capabilities was observed in a wide range of about 60-120 min after lunch ${ }^{(1,4,13)}$. In our previous studies, cognitive parameters were measured $45 \mathrm{~min}$ after lunch, without any evidence of a post-lunch dip. It remains an open question whether a meal-enhanced post-lunch dip in children exists as has been suggested for adults. As a post-lunch dip was mostly seen in sustained attention tasks ${ }^{(21,22)}$, it might especially be detectable in the alertness task (which includes testing for sustained attention). As our results did not show a significant difference between the $\mathrm{L}$ and the NL condition, it could be assumed that there might not be a post-lunch dip in children. However, to answer this question conclusively, further studies will be needed.

There are several characteristics of the CoCo study design that need to be discussed. The study was not conducted under clinical conditions, but tested the students in real-life conditions in their classroom setting. Accordingly, factors apart from lunch 
such as environmental stress (e.g. noise, peer group actions) might have influenced individual cognitive performance ${ }^{(23)}$. Especially auditory distraction can have detrimental effects ${ }^{(24,25)}$ on the cognitive performance in children. Even though the study team tried to keep the children in the testing room as quiet as possible, environmental disturbances could partly have masked acute individual effects of lunch. Clinical studies might be more suitable to clearly identify isolated lunch effects, but do not allow any conclusions on the practical meaning of these results in children's everyday life. In addition, the cross-over design of the CoCo study should have minimised potential effects of individual confounding in the total sample. To counteract a possible learning effect, we used a parallel version of the cognitive task in which the task switching and the twoback sequences differed (task switching task differed in position of the items, two-back task in sequence of fruit and vegetable items). In addition, we conducted a training phase immediately before the actual testing to ensure that every subject understands the tests already before the first assessment. The taskswitching task and the two-back task, which were already used in our previous study, were originally designed for adults. Although we adapted these tests for children and tested the entire test battery in a pretest with children of the same age in another school in order to avoid a very sophisticated test, the rate of implausible two-back test results might indicate a floor effect in the same subjects.

In the CoCo study, no pre-lunch performance was measured as opposed to laboratory studies. However, we decided against this practice as we were worried about potential negative effects on the motivation with increasing numbers of tests. If we had included a pre-lunch measurement, the children would have had to complete four tests on 2 test days within a week, and without a pre-lunch testing only two. Negative influences on motivation might not only have impact on the results of the cognitive tasks, but could also result in a high rate of dropouts. Therefore, we decided, for this study, to focus on the afterlunch condition.

Another limitation of the CoCo study is that it was not possible to use a double-blind design with a placebo condition. This leaves the possibility of subject and experimenter bias and is a common problem in food-based trials. However, the randomised, cross-over design eliminates variations between the subjects and reduces bias. In the present study, fasting was the control condition. However, fasting could also be viewed as intervention as the majority of students regularly eat lunch at school. In a recent review, which compared ten studies of adults for the impact of short-term fasting on cognition ${ }^{(26)}$, results were equivocal. Although some studies showed no effects of fasting on cognition, others showed impairments in tasks related to psychomotor speed, mental rotation or executive function. However, these results are not transferable to the current study because of differences in cognitive tasks, fasting periods, time of fasting, time of day and the age of the participants.

Müller et $a l^{(11)}$ concluded that lunch effects in studies of adults might have been modified by the fact that the test meal size was larger or smaller than the usual lunch size. Although the test meal size was assessed in the CoCo study, it was not possible to assess probable differences with respect to the individual usual lunch size as well. Hence, future studies on cognitive effects of lunch should assess the usual size of lunch to examine the impact of deviation of the test lunch from habitual eating lunch size (smaller or larger) on cognitive performance.

A considerable number of participants did not completely comply with the study protocol ( $n$ 67). Reasons for this behaviour were not enquired, but it could be assumed that it was difficult for the children to restrain from eating during this time period, especially when it was explicitly forbidden. Apart from the questionnaire at the end of the test day and supervision in the school yard, children had opportunities for hidden snacking. Thus, it could not be fully ruled out that all participants who did not comply with the protocol were detected. In addition, it has to be mentioned that the sixth grade students of this intervention already participated as fifth grade students in the previous intervention. Therefore, they may have been familiar with two of the three cognitive tasks and were probably less motivated. However, any such effects should have been minimised as the differences between the test days were analysed in the cross-over design.

In conclusion, the present study confirmed the results of our previous studies ${ }^{(11,12)}$ that school lunch does not seem to impair children's CF regarding the conducted tests on task switching, working memory updating and alertness. Although the previous studies $^{(11,12)}$ pointed to slight improvements in single cognitive parameters by lunch shortly after the meal, the current study did not indicate positive effects of lunch on cognition after a prolonged interval until early afternoon.

\section{Acknowledgements}

The authors thank the children and their teachers for their participation and the kitchen staff for supporting this study. The authors acknowledge the dedicated fieldwork staff. Particular thanks to Ludger Blanke (Institute of Working, Aging and Learning (ALA) Institute) for advice on application and evaluation of the cognition test battery and the excellent technical support.

CogniDo Continued (CoCo) was supported by a grant from the Uniscientia Foundation, Vaduz, Liechtenstein. Uniscientia had no role in the design, analysis or writing of this article.

The authors' contributions are as follows: L. L., M. K. and M. S. formulated the specific research question and the design of the study. M. F. designed the test battery for cognitive testing and helped with interpreting the results of cognitive testing. The study was carried out and data were analysed by M. S. and L. L. The article was written by M. S. and L. L. with input from K. M., M. F., M. K. and P. S. The final manuscript was read and approved by all the authors.

The authors declare that there are no conflicts of interest.

\section{References}

1. Craig A, Baer K \& Diekmann A (1981) The effects of lunch on sensory-perceptual functioning in man. Int Arch Occup Environ Health 49, 105-114. 
2. Smith AP \& Miles C (1986) Effects of lunch on selective and sustained attention. Neuropsychobiology 16, 117-120.

3. Kanarek RB \& Swinney D (1990) Effects of food snacks on cognitive performance in male college students. Appetite 14, 15-27.

4. Smith AP \& Miles C (1986) The effects of lunch on cognitive vigilance tasks. Ergonomics 29, 1251-1261.

5. Monk TH (2005) The post-lunch dip in performance. Clin Sports Med 24, e15-e23 xi-xii.

6. Pollitt E, Cueto S \& Jacoby ER (1998) Fasting and cognition in well- and undernourished schoolchildren: a review of three experimental studies. Am J Clin Nutr 67, 779S-784S.

7. Hoyland A, Dye L \& Lawton CL (2009) A systematic review of the effect of breakfast on the cognitive performance of children and adolescents. Nutr Res Rev 22, 220-243.

8. Adolphus K, Lawton CL \& Dye L (2013) The effects of breakfast on behavior and academic performance in children and adolescents. Front Hum Neurosci 7, 425.

9. Bellisle F (2004) Effects of diet on behaviour and cognition in children. Br J Nutr 92, Suppl. 2, S227.

10. Müller K, Libuda L, Terschlüsen AM, et al. (2013) A review of the effects of lunch: on adults' short-term cognitive functioning. Can J Diet Pract Res 74, 181-188.

11. Müller K, Libuda L, Gawehn N, et al. (2013) Effects of lunch on children's short-term cognitive functioning: a randomized crossover study. Eur J Clin Nutr 67, 185-189.

12. Schröder M, Müller K, Falkenstein M, et al. (2015) Short-term effects of lunch on children's executive cognitive functioning: the randomized crossover Cognition Intervention Study Dortmund PLUS (CogniDo PLUS). Physiol Behav 152, 307-314.

13. Craig A (1986) Acute effects of meals on perceptual and cognitive efficiency. Nutr Rev 44, Suppl, 163-171.

14. Tukey JW (1977) Exploratory Data Analysis. Vol. 1: Quantitative Methods, Reading. Massachusetts, MA: Addison-Wesley.

15. Grizzle JE (1965) The two-period change-over design and its use in clinical trials. Biometrics 21, 467.
16. Donohoe RT \& Benton D (1999) Cognitive functioning is susceptible to the level of blood glucose. Psychopharmacology 145, 378-385.

17. Owen L, Scholey AB, Finnegan Y, et al. (2012) The effect of glucose dose and fasting interval on cognitive function: a double-blind, placebo-controlled, six-way crossover study. Psychopharmacology 220, 577-589.

18. Benton D, Brett V \& Brain PF (1987) Glucose improves attention and reaction to frustration in children. Biol Psychol 24, 95-100.

19. Wang SR, Chase HP, Garg SK, et al. (1991) The effect of sugar cereal with and without a mixed meal on glycemic response in children with diabetes. I Pediatr Gastroenterol Nutr 13, 155-160.

20. Buss RW, Kansal PC, Roddam RF, et al. (1982) Mixed meal tolerance test and reactive hypoglycemia. Horm Metab Res $\mathbf{1 4}$ 281-283.

21. Craig A \& Richardson E (1989) Effects of experimental and habitual lunch-size on performance, arousal, hunger and mood. Int Arch Occup Environ Heath 61, 313-319.

22. Smith A, Ralph A \& McNeill G (1991) Influences of meal size on post-lunch changes inperformance efficiency, mood, and cardiovascularfunction. Appetite 16, 85-91.

23. Isaacs E \& Oates J (2008) Nutrition and cognition: assessing cognitive abilities in children and young people. Eur J Nutr 47, Suppl. 3, 4-24.

24. Beaman CP (2005) Auditory distraction from low-intensity noise: a review of the consequences for learning and workplace environments. Appl Cogn Psychol 19, 1041-1064.

25. Elliott EM (2002) The irrelevant-speech effect and children: theoretical implications of developmental change. Mem Cognit 30, 478-487.

26. Benau EM, Orloff NC, Janke EA, et al. (2014) A systematic review of the effects of experimental fasting on cognition. Appetite 77, 52-61. 